Tropical Journal of Pharmaceutical Research October 2013; 12 (5): 691-698

ISSN: $1596-5996$ (print); 1596-9827 (electronic) (C) Pharmacotherapy Group, Faculty of Pharmacy, University of Benin, Benin City, 300001 Nigeria.

\title{
Plumbagin Inhibits Leptin-Induced Proliferation of Hepatic Stellate Cells via JAK2-STAT3 Pathway to Protect against Hepatic Fibrosis
}

\author{
Yanfei Wei ${ }^{1}$, Tiejian Zhao ${ }^{{ }^{*}}$, Zhiwei Zhang ${ }^{2}$, Xuemei Liu ${ }^{1}$, Zhenqing Huang ${ }^{1}$, \\ Yuan Zhang ${ }^{1}$ and Jingqiang $\mathrm{Li}^{1}$ \\ ${ }^{1}$ Department of Physiology, Guangxi University of Chinese Medicine, 179\# East Mingxiu Road, Nanning, Guangxi, 530001, \\ ${ }^{2}$ Ruikang Hospital, Guangxi University of Chinese Medicine, Nanning, 10\#, Huadong Road, Guangxi 530021, PR China. \\ *For correspondence: Email: zhaotiejian@163.com; Tel: +86-771-2214279; Fax: +86-771-2214279
}

Revised accepted: 7 July 2013

\begin{abstract}
Purpose: To investigate the protective effects of plumbagin against liver fibrosis and explore the influence of plumbagin on the proliferation of hepatic stellate cells (HSCs).

Methods: HSC-LX2 cells were divided into blank/control group, $100 \mathrm{ng} / \mathrm{ml}$ leptin group, $100 \mathrm{ng} / \mathrm{ml}$ leptin +2 $\mu \mathrm{mol} / \mathrm{L}$ plumbagin group, $100 \mathrm{ng} / \mathrm{ml}$ leptin $+8 \mu \mathrm{mol} / \mathrm{L}$ plumbagin group and $100 \mathrm{ng} / \mathrm{ml}$ leptin $+6.25 \mu \mathrm{g} / \mathrm{ml}$ colchicines group. The expressions of leptin receptor protein (OB-Rb), p-JAK2, p-STAT3, JAK2, STAT3, p$E R K 1 / 2$, ERK1/2 and MMP-1 were detected by Western blot assay. The content of type I collagen in the supernatant of HSCs was also measured after stimulation by leptin.

Results: Leptin induced OB-Rb expression in HSCs, which reached a peak level at $24 \mathrm{~h}(\mathrm{p}<0.01)$. Leptininduced $O B-R b$ expression was significantly inhibited by plumbagin at concentrations of $2 \mu m o l / L$ and 8 $\mu \mathrm{mol} / \mathrm{L}$, respectively. Western blot assay revealed that plumbagin significantly decreased pJAK2 expression in leptin-treated HSCs $(p<0.01)$. Leptin induced expression of pSTAT3 significantly decreased after plumbagin treatment in HSC-LX2 $(p<0.01)$. $p$-ERK1/2 expression markedly decreased in plumbagin-treated HSCs $(p<0.01)$. Plumbagin significantly increased MMP-1 expression in leptin-treated HSCs $(p<0.01)$.

Conclusion: Plumbagin has an anti-fibrotic effect and may decrease the protein expressions of components in JAK2-STAT3 pathway to inhibit HSC proliferation. Thus, plumbagin may be useful in the clinical prevention and treatment of liver fibrosis.
\end{abstract}

Keywords : Plumbagin, Leptin, Hepatic stellate cell, Proliferation, Signal transduction pathway, Antiinflammatory

Tropical Journal of Pharmaceutical Research is indexed by Science Citation Index (SciSearch), Scopus, International Pharmaceutical Abstract, Chemical Abstracts, Embase, Index Copernicus, EBSCO, African Index Medicus, JournalSeek, Journal Citation Reports/Science Edition, Directory of Open Access Journals (DOAJ), African Journal Online, Bioline International, Open-J-Gate and Pharmacy Abstracts

\section{INTRODUCTION}

Hepatic fibrosis, characterized by abnormal accumulation of extracellular matrix (ECM), is a wound-healing process responsive to a variety of factors, such as cytokines, growth factors, and inflammatory mediators. Previous studies have showed that ECM is mainly derived from hepatic stellate cells (HSCs) in the process of hepatic fibrosis[1,2]. Activation of resident HSCs into proliferative, contractile, and fibrogenic cells is a dominant cause of liver injury.

Recently, evidence suggests a critical role of leptin in the hepatic inflammation and fibrogenesis [3]. When chronic liver injury occurs, leptin augments net production of extracellular 
matrix (ECM) by increasing HSC proliferation, elevating type I collagen synthesis and tissue inhibitor of metalloproteinase 1 (TIMP-1), and strongly inhibiting the apoptosis of HSCs [4, 5]. In HSCs, leptin binds to the long form leptin receptor, Ob-Rb, and activates Janus kinase 2(JAK2)/signal transducer and activator of transcription 3 (STAT3) pathway, leading to the enhanced synthesis and secretion of collagen and increased ECM deposition. In recent years, a large number of clinical and experimental studies have demonstrated that leptin is closely related to liver fibrosis.

This study was undertaken to investigate the effect of plumbagin on the activation of signal molecules in HSCs. Our finding may elucidate the potential mechanism underlying the protective effect of plumbagin on hepatic fibrosis and uncover more targets for inhibiting leptininduced HSC activation, thus possibly providing evidence and method for clinical prevention and treatment of hepatic fibrosis.

\section{EXPERIMENTAL}

\section{Cell lines, reagents and antibodies}

LX-2 cells, an immortalized human hepatic stellate cell line, were provided by the Medical Experimental Center of Xiangya Medical College. Plumbagin was purchased from Sigma. Recombinant leptin was obtained from Protech. Rabbit monoclonal anti-OB-R antibody was from Bioworld (USA). Rabbit polyclonal anti-JAK2 antibody and rabbit polyclonal anti-phosphoJAK2 antibody were from Bioss (USA). Rabbit monoclonal anti-STAT3, anti-phospho-STAT3, anti-ERK1/2, anti-phospho-ERK1/2, anti-MMP-1, anti-MMP-13 and anti-collagen antibodies were obtained from Bioworld (UK).

\section{Enzyme-linked immunosorbent assay (ELISA)}

In brief, $2 \mathrm{~mL}$ of HSCs suspension was seeded into 6-well plates at $1 \times 10^{5}$ cells $/ \mathrm{mL}$ and cultured at $37{ }^{\circ} \mathrm{C}$ in a humidified atmosphere with $5 \%$ $\mathrm{CO}_{2}$ for $2 \mathrm{~h}$. Cells were treated with $100 \mathrm{ng} / \mathrm{mL}$ leptin, $100 \mathrm{ng} / \mathrm{mL}$ leptin plus $2 \mu \mathrm{mol} / \mathrm{L}$ plumbagin, $100 \mathrm{ng} / \mathrm{mL}$ leptin plus $8 \mu \mathrm{mol} / \mathrm{L}$ plumbagin, 100 $\mathrm{ng} / \mathrm{mL}$ leptin plus $6.25 \mathrm{~g} / \mathrm{ml}$ colchicine, respectively. HSCs without treatment served as the negative control group, and those treated with $100 \mathrm{ng} / \mathrm{mL}$ leptin plus colchicine as the positive control group., The content of type I collagen in the supernatant of HSCs was measured by ELISA $24 \mathrm{~h}$ later.

\section{Cell culture}

HSC-LX2 cells were incubated in high-glucose Dulbecco's Modified Eagle Medium (DMEM) supplemented with $2 \mathrm{mM}$ L-glutamine, $100 \mathrm{U} / \mathrm{mL}$ penicillin, $100 \mu \mathrm{g} / \mathrm{mL}$ of streptomycin, $50 \mu \mathrm{M} 2$ Mercaptoethanol (2-ME), and $10 \%$ fetal calf serum (FCS; all from Life Technologies, Invitrogen, USA) at $37{ }^{\circ} \mathrm{C}$ in a humidified incubator with $5 \% \mathrm{CO}_{2}$. Cells were trypsinized, washed and cultured in 6-well plates (Corning, $\mathrm{NY})$ at $5 \times 10^{4}$ cells $/ \mathrm{mL}$ in duplicate. After $24 \mathrm{~h}$, leptin was added to corresponding wells and the expressions of target proteins were detected at $0,6,12,24,36$ and $48 \mathrm{~h}$.

\section{SDS-PAGE and Western blot assay}

Western blot assay was performed as described before [6]. Cells were collected and washed twice with cold phosphate buffered saline (PBS). Then, these cells were lysed with radioimmunoprecipitation assay (RIPA) Lysis Buffer (50 mmol/L Tris, $1 \% \mathrm{NP}-40,150 \mathrm{mmol} / \mathrm{L} \mathrm{NaCl}, 1$ $\mathrm{mmol} / \mathrm{L}$ ethylenediaminetetraacetic acid (EDTA), $0.1 \%$ SDS). The proteins were quantified using the Bradford method. Proteins were subjected to sodium dodecyl sulfate polyacrylamide gel electrophoresis (SDS-PAGE) and then transferred electrophoretically onto a nitrocellulose membrane. The membrane was blocked with $5 \%$ non-fat milk in Tris Buffered Saline with Tween (TBST) $(50 \mathrm{mmol} / \mathrm{L}$ Tris- $\mathrm{HCl}$, $150 \mathrm{mmol} / \mathrm{L} \mathrm{NaCl}$ and $0.1 \%$ Tween), and then incubated with rabbit monoclonal antibody against OB-Rb, phosphor-JAK2, phosphorSTAT3, JAK2, STAT3, phosphor-ERK1/2, ERK1/2, MMP-1, MMP-13 or gyceraldehyde 3phosphate dehydrogenase(GAPDH). The goat anti-rabbit IgG antibody conjugated to horseradish peroxidase was used as a secondary antibody. The resulting bands were detected using an enhanced chemiluminescence detection system according to the manufacturer's instructions. GAPDH was used as an internal reference and three independent experiments were performed.

\section{Data analysis}

All experimental data were analyzed by SPSS version 13.0 and expressed as mean \pm standard deviation (SD). Data among groups were compared using analysis of variance and those between groups by Student $t$ test. A value of $p<$ 0.05 was considered statistically significant. Detection of optical density of each band and image analyses were performed using Quantity One 4.62 analysis software. 


\section{RESULTS}

\section{Effect of leptin and plumbagin on OB-Rb expression}

As shown in Figure $1 \mathrm{~A}$, leptin induced $\mathrm{OB}-\mathrm{Rb}$ expression in HSCs, which reached a peak level at $24 \mathrm{~h} \quad(P<0.01) \quad$ (Figure $1 \mathrm{C})$. The OB-Rb expression increased in a time dependent manner within $36 \mathrm{~h}$. However, OB-Rb expression gradually decreased to baseline level at $48 \mathrm{~h}$.

Based on the above results, cells were then treated with $100 \mathrm{ng} / \mathrm{mL}$ leptin for $24 \mathrm{~h}$ to induce $\mathrm{OB}-\mathrm{Rb}$ expression. As shown in Figure $1 \mathrm{~B} \& \mathrm{D}$, leptin induced $\mathrm{OB}-\mathrm{Rb}$ expression was significantly inhibited by plumbagin at $2 \mu \mathrm{mol} / \mathrm{L}$ and $8 \mu \mathrm{mol} / \mathrm{L}$. Untreated cells were used as a negative control group and cells treated with 100 $\mathrm{ng} / \mathrm{mL}$ leptin alone as a positive control group.

\section{Effect of leptin and plumbagin on JAK2 activation in HSC-LX2}

As shown in Figures $2 \mathrm{~A}$ and $\mathrm{B}$, phosphorylated JAK2 was detected at different time points after leptin treatment. After leptin treatment for $6 \mathrm{~h}, \mathrm{p}$ JAK2 expression in HSCs increased $(p<0.05)$, and reached a peak level at $24 \mathrm{~h}(p<0.01)$. Thereafter, p-JAK2 expression decreased

A

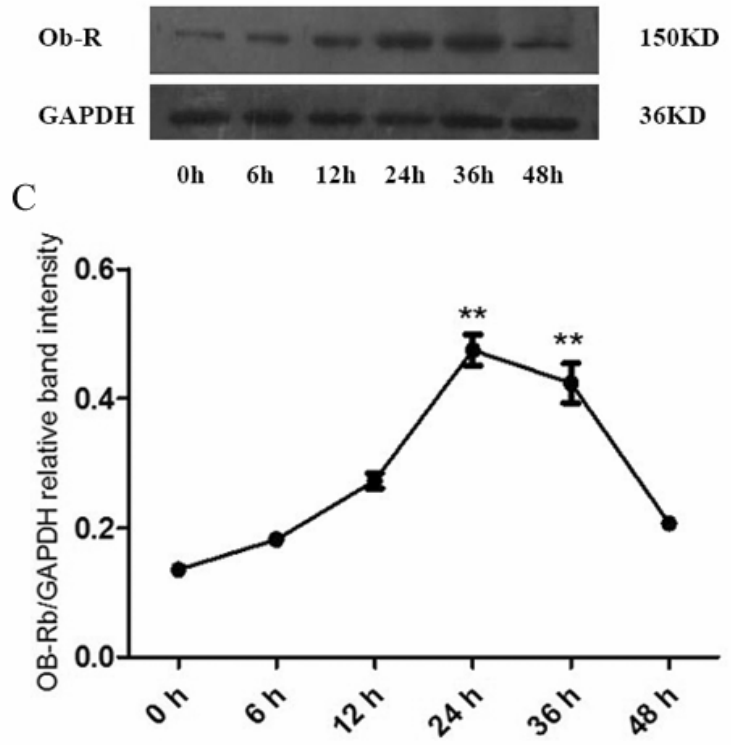

gradually and was similar to the baseline level at $48 \mathrm{~h}$. In the study, cells treated with leptin for 24 $\mathrm{h}$ were used as a positive control group. Western blot assay revealed that plumbagin significantly decreased the pJAK2 and JAK2 expression in leptin treated HSCs $(p<0.01)$, as shown in Figure $2 \mathrm{C}$ and $\mathrm{D}$.

\section{Effect of leptin and plumbagin on STAT3 and pSTAT3 expressions in HSC-LX2}

STAT3 plays a key role in many cellular processes such as cell growth and apoptosis, and mediates the expression of a variety of genes in response to stimuli including leptin. Thus, the relationship between plumbagin and leptin induced expressions of STAT3 and pSTAT3 were evaluated in HSC-LX2. As shown in Fig. $3 A$ and B, p-STAT3 expression in HSCs was enhanced after treatment with leptin for $6 \mathrm{~h}$, and reached a peak level at $12 \mathrm{~h}(p<0.01)$. Subsequently, p-STAT3 expression decreased, and was close to the baseline level at $48 \mathrm{~h}$. As expected, plumbagin inhibited the expressions of STAT3 and PSTAT3 in leptin-treated HSC-LX2. As shown in Fig. $3 C$ and D, leptin induced expressions of STAT3 and PSTAT3 were significantly decreased after plumbagin treatment in HSC-LX2.

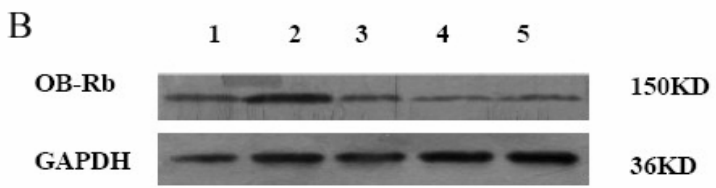

D

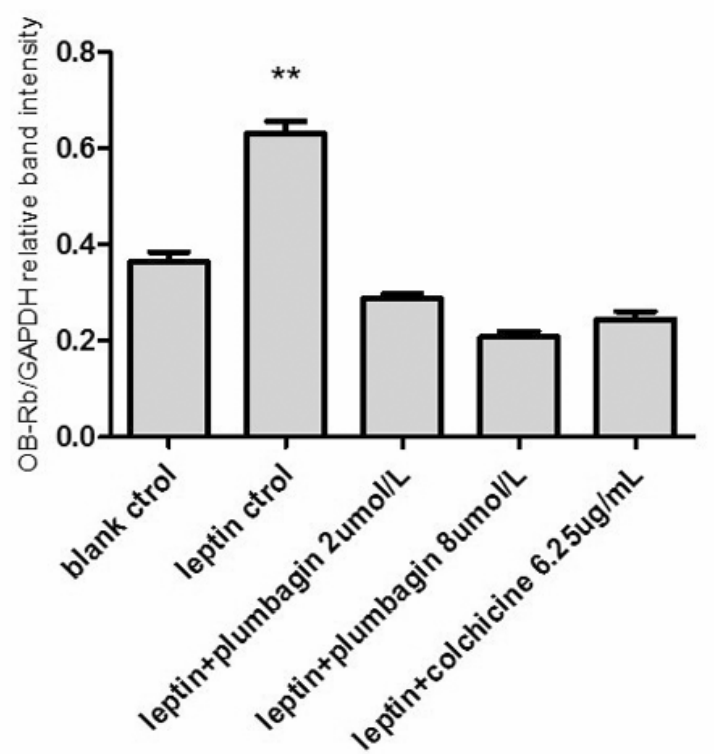

Figure 1: OB-Rb expression in HSC-LX2. $A=$ Western blot assay result; $\mathrm{C}=$ relative band expression of $\mathrm{OB}-\mathrm{Rb}$ and GAPDH at different time points; $B=$ Western blot assay after plumbagin treatment; $D=$ relative band expressions of OB-Rb and GAPDH after plumbagin treatment; ${ }^{* \star} p<0.01$ vs blank control. 
A
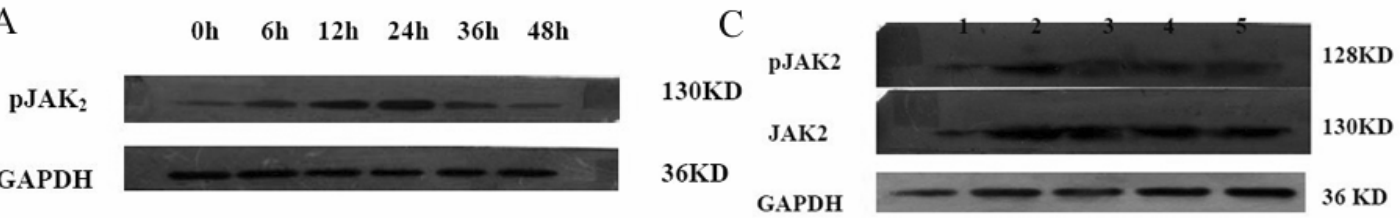

B



D
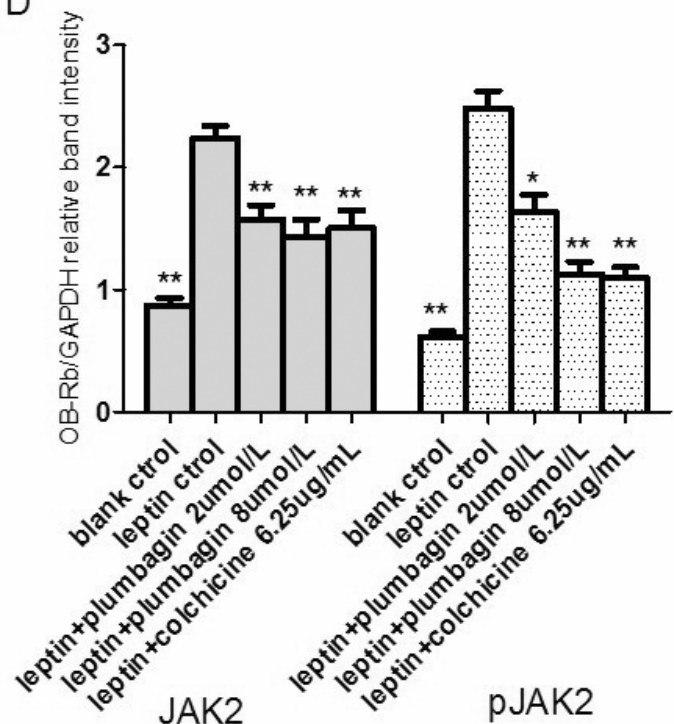

Figure 2: Expressions of JAK2 and pJAK2 in HSC-LX2. $A=$ Western blot assay of pJAK2; $B=$ Expressions of pJAK2 and GAPDH at different time points; $C=$ Western blot assay of JAK2 and pJAK2 after plumbagin treatment; $\mathrm{D}=$ plumbagin treatment significantly reduced pJAK2 and JAK2 expression $(p<0.01)$; for Figure 2B, ${ }^{\star} P<0.05,{ }^{* *} p<0.01$ vs $0 \mathrm{~h}$; for Figure $2 \mathrm{D},{ }^{*} p<0.01,{ }^{* *} p<0.01$ vs cells treated with leptin alone.

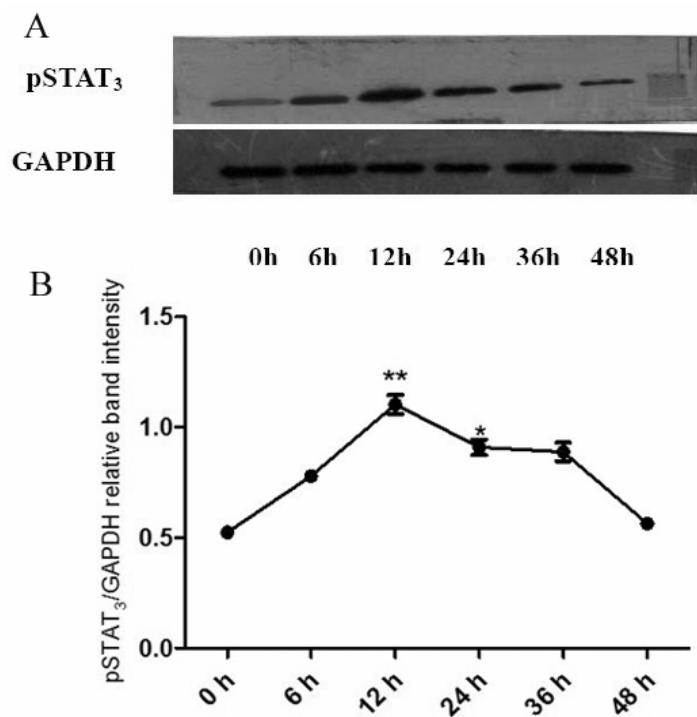

$\mathrm{C}$ $92 \mathrm{KD} \quad \mathrm{pSTAT}_{3}$ $36 \mathrm{KD} \quad \mathrm{STAT}_{3}$

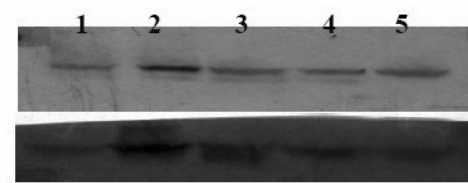
92KD GAPDH 89KD $\mathrm{D}$

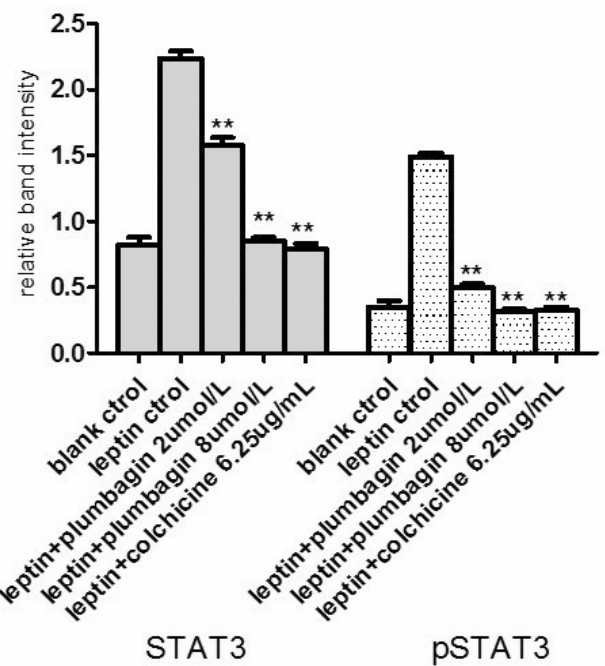

Figure 3: Expressions of STAT3 and pSTAT3 receptor protein in HSC-LX2: A, Western blot assay of pSTAT3; B, Expressions of pSTAT3 and GAPDH at different time points; C, Expressions of STAT3, and pSTAT3 receptor protein after plumbagin treatment; $D$, Plumbagin treatment significantly decreased protein expressions of STAT3 and pSTAT3 receptor; for Figure $3 \mathrm{~B},{ }^{*} p<0.05$, ${ }^{* *} p<0.01$, vs $0 \mathrm{~h}$; for Figure $3 \mathrm{D},{ }^{*} p<0.05,{ }^{* *} p<0.01$ vs cells treated with leptin alone. 

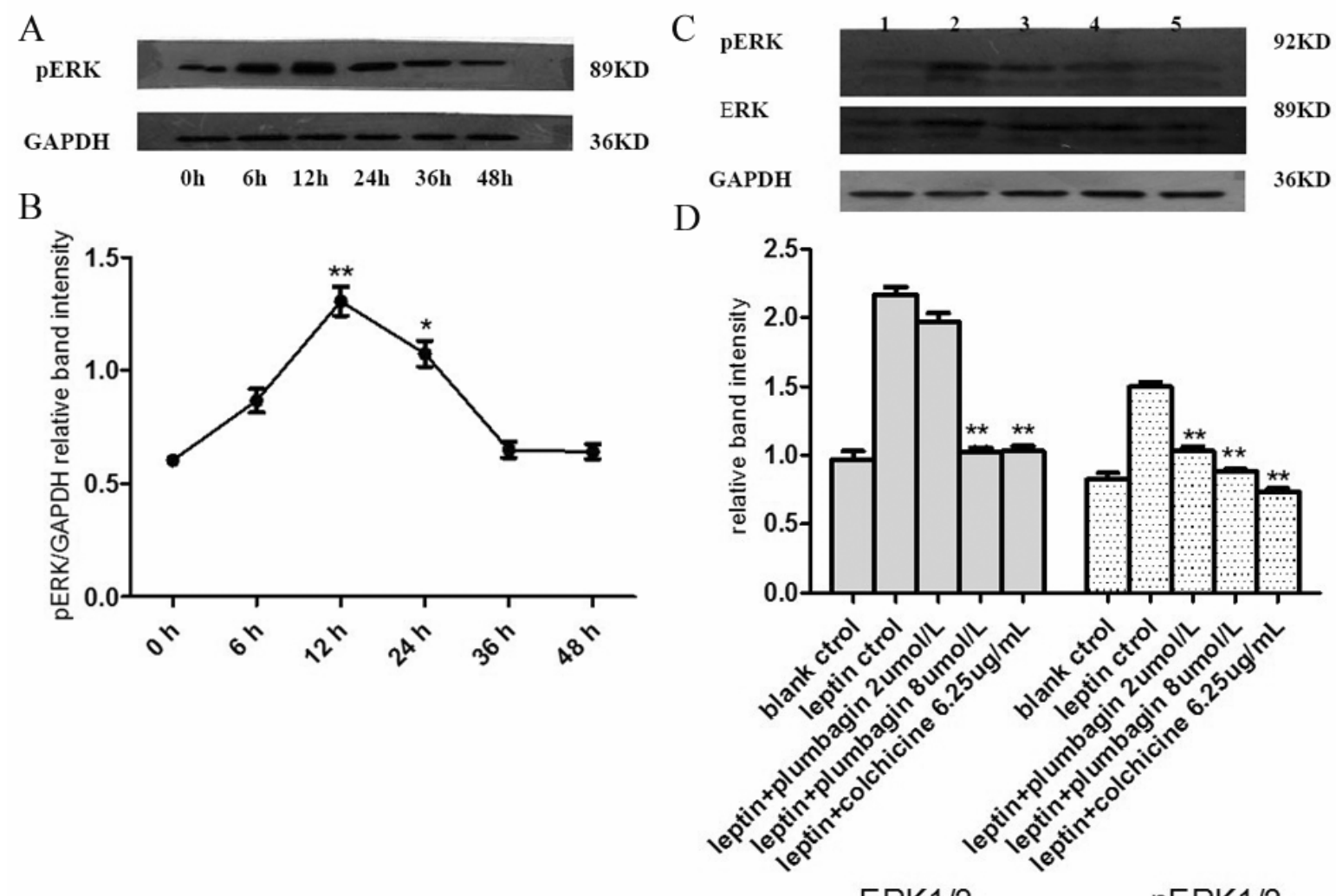

$\mathrm{ERK} 1 / 2$

$\mathrm{pERK} 1 / 2$

Figure 4: Expressions of ERK and pERK in HSC-LX2: $A=$ Western blot assay of pERK; $B=$ expressions of pERK and GAPDH at different time points; $C=$ expressions of ERK and pERK after plumbagin treatment; $D=$ plumbagin treatment significantly reduced protein expressions of ERK1/2 and pERK1/2; for Figure 4B, ${ }^{*} p<0.05$, ${ }^{\star *} p<0.01$ vs $0 \mathrm{~h}$; for Figure $4 \mathrm{D},{ }^{*} p<0.05,{ }^{\star *} p<0.01$ vs cells treated with leptin alone.

\section{Effect of plumbagin on ERK1/2 and pERK1/2 activation in leptin-treated HSC-LX2}

ERK $1 / 2$ are members of the mitogen-activated protein kinase (MAPK) superfamily that can translocate into the nucleus and regulate cell proliferation and apoptosis. As shown in Fig.4A\&B, pERK1/2 expression significantly increased in HSC-LX2 after leptin treatment, and p-ERK1/2 expression reached a peak level at 12 h. However, the $\mathrm{p}$-ERK $1 / 2$ expression markedly decreased in plumbagin-treated HSCs (Figure $4 C$ and $D)$.

\section{Plumbagin increased MMP-1 production in leptin treated HSC-LX2}

MMP-1 is also known as interstitial collagenase and fibroblast collagenase and an enzyme encoded by MMP1 gene in humans. As shown in Fig $5 \mathrm{~A}$ and $\mathrm{B}$, the MMP-1 expression in HSCs dramatically decreased after leptin treatment, but plumbagin significantly increased MMP-1 expression in these leptin treated HSCs. However, no changes were observed in MMP-13 expression as shown in western blot assay.

\section{Plumbagin decreased type I collagen content of leptin-treated HSC-LX2}

The production of type I collagen was also measured in the supernatant of HSC-LX2. As shown in Figure $5 C$ and $D$, when compared with untreated cells, the content of type I collagen in leptin-treated HSC increased significantly $(p<$ 0.01 ). However, plumbagin markedly inhibited the expression and secretion of type I collagen in leptin treated HSCs.

\section{DISCUSSION}

In 1994, Zhang et al [7] discovered the mouse obese gene and its human homologue by positional cloning for the first time. Shimamura et al [8] synthesized its protein product leptin encoded by human and mouse obese gene using recombinant DNA technology. The discovery of leptin is a major breakthrough in the studies on obesity and energy metabolism. Leptin, a $16-\mathrm{KDa}$ hormone, is secreted mainly by adipocytes and acts on the hypothalamus to control body weight by reducing food intake and increasing energy consumption [9]. Recently, some experiments and clinical studies have shown that leptin is closely related to liver fibrosis 
A

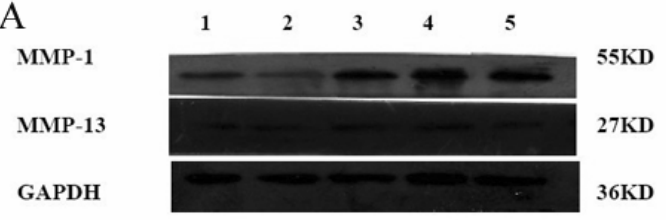

B

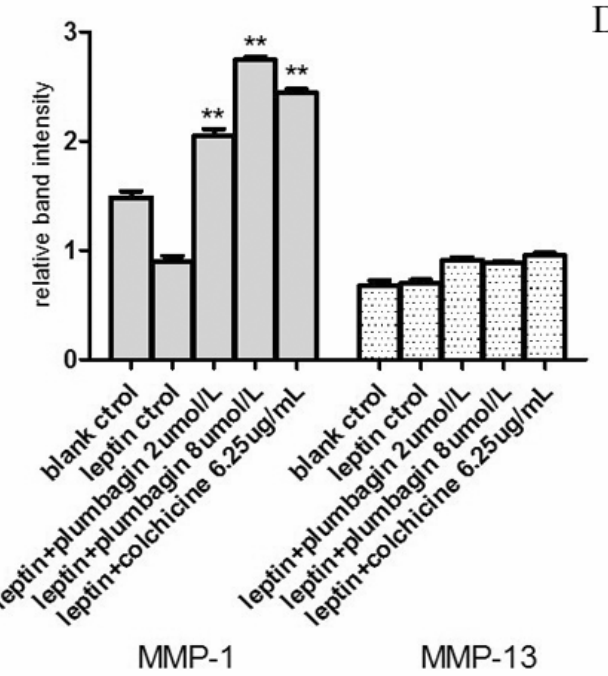

$\mathrm{C}$

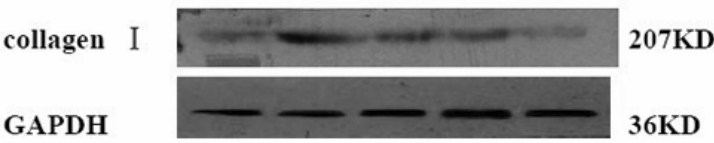

$\mathrm{D}$

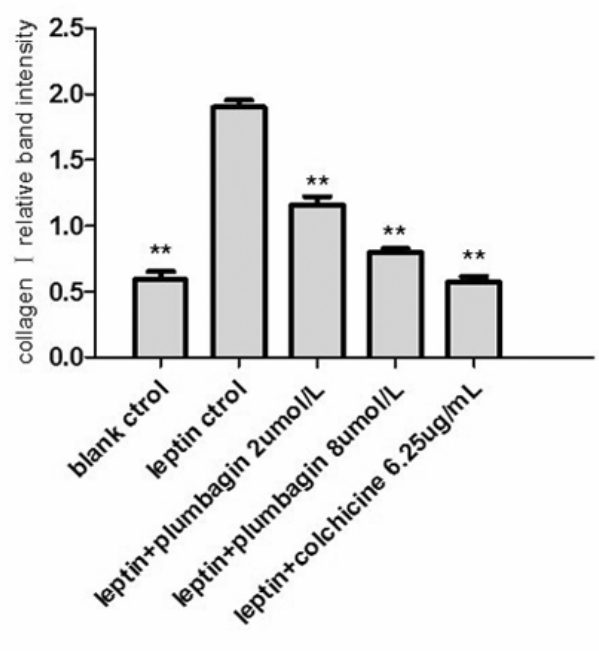

Figure 5: Expressions of MMP-1, MMP-13 and collagen I in HSC-LX2: A = Western blot assay of MMP-1 and MMP-13; $\mathrm{B}=$ relative expression of MMP-1, MMP-13 MMP-13 expression remained unchanged $(p<0.01) ; \mathrm{C}=$ expression of collagen I and GAPDH. D = relative expression of collagen I after plumbagin treatment; ${ }^{*} p<0.01$, ${ }^{* *} p<0.01$ vs cells treated with leptin alone.

and cirrhosis, and it plays a key role in the development of liver fibrosis via inducing HSC activation and proliferation.

Leptin has characteristics of some cytokines when exerting biological effects, and its biological effects require its binding to leptin receptor (OB$\mathrm{R})$. OB-R is a member of the class I cytokine receptor family, and is composed of a single subunit containing extracellular domain, transmembrane domain and intracellular domain[10,11]. OB-R contains a long chain and a short chain, and its biological effects are attributed to the activation of the long chain. Leptin-induced HSC proliferation is related to the activation of JAK-STAT, MAPK and P13K/AKT and on the subsequent activation of signal transduction pathways[12,13]. JAK/STAT signaling is associated with physiological and pathological responses, involved in cell proliferation, differentiation, functioning and apoptosis, and is an important pathway mediating cytokine signal transduction[14,15].

JAK-STAT pathway is an important pathway related to leptin induced HSC proliferation. OB-R lacks intrinsic kinase activity and may mediate multiple signaling pathways by binding to cytoplasmic kinases such as Janus Kinase 2 (JAK2). Activation of JAK2 by leptin promotes tyrosine phosphorylation of OB-R at Tyr-986, Tyr-1078 and Tyr-1141, thus activating OB-R. Activation of OB-R by leptin activates signaling modules such as JAK/STAT module. Tyrosine phosphorylation of OB-R induces the binding of STATs to OB-R. Binding of STATs to phosphorylated OB-R leads to JAK2 mediated tyrosine phosphorylation and STAT activation. Activated STATs translocate to the nucleus to induce gene expressions, ultimately exerting biological effects[16,17].

In vitro studies have found that exogenous leptin may significantly stimulate HSC activation [18], proliferation and phenotypic change, paralleling with type I collagen synthesis. Its activation is mainly associated with the activation of signal transduction, transcriptional activation factor, mitogen-activated protein kinase and phosphoinositide-3 kinase. Leptin may promote HSC activation and collagen synthesis in these cells via activating MAPK signaling pathways. In eukaryotic cells, 4 MAPK pathways have been identified: ERK1/2, c-jun, p38 and ERK5 pathways, which play important roles in cell growth, development and proliferation. Upon activating, ERK enters the nucleus, and phosphorylates several transcriptional factors (such as c-Myc, ELK, c-jun and c-fos) to regulate gene expression [19]. After coupling and

Trop J Pharm Res, October 2013;12 (5): 6 
activation of JAK tyrosine protein kinase, ERK may promote cell proliferation through activating ERK1/2 and p38, resulting in hepatic fibrosis [19].

At the early stage of carbon tetrachloride-induced hepatic fibrosis, the serum leptin concentration is significantly elevated in rats. Due to the key role of leptin in the development of liver fibrosis, liver fibrosis may be treated by inhibiting or blocking leptin activation in HSCs. Our preliminary experiments have shown that plumbagin and its active ingredients possess protective effects on carbon tetrachloride induced liver fibrosis in rats. Plumbagin may reduce TGF- $\beta 1$ in liver fibrosis of rats, significantly inhibit the proliferation of HSCT6 cells in vitro, and suppress the expressions of transforming growth factor $\beta 1$ and $\alpha$-smooth muscle actin. Many studies have shown that the Chinese herbal compound may exert pharmacological anti-fibrotic effects at multiple levels and multiple targets.

The present study attempts to explore the mechanism underlying the anti-fibrotic effect of plumbagin at the receptor level. There is evidence showing that leptin may affect the expression of leptin receptor in hepatocytes and influence its phosphorylation. Leptin and its receptor may exert a positive regulatory feedback, which enables a smoother leptin signal transduction and may be better for exerting their biological effects [20]. Results from our experiments showed that, leptin at optimal concentration could induce leptin receptor expression in HSCs in a time dependent manner, which reached a peak level at $24 \mathrm{~h}$. After plumbagin treatment, the OB-Rb expression in HSCs was significantly reduced, indicating that plumbagin blocks leptin at the receptor level.

The effect of the Chinese herb has multiple targets, and thus we hypothesize whether the antifibrotic effect of plumbagin is related to the blocking of post-receptor signal transduction pathways, namely the cytoplasmic or nuclear signal transduction process in HSCs. In the following experiments, our results showed that plumbagin directly blocked the expressions of JAK2 and STAT3, the two components of JAKSTAT signaling pathway. Compared with blank control group, leptin significantly increased the protein expressions of JAK2, pJAK2, STAT3 and pSTAT3. Compared with cells treated with leptin alone, plumbagin significantly decreased protein expressions of pJAK2, JAK2, STAT3 and pSTAT3. This suggests that plumbagin inhibits the STAT3 activation at JAK2 phosphorylation level, and may inhibit HSC proliferation through JAK-STAT signaling pathway. In addition, leptin significantly increased ERK $1 / 2$ and $p E R K 1 / 2$ protein expressions, whereas plumbagin markedly decreased them, indicating that plumbagin also blocks ERK phosphorylation in the leptin-dependent MAPK signaling pathway, significantly inhibiting the proliferation of HSCs. As liver fibrosis occurs, the number of HSCs markedly increases, and they undergo a phenotypic change into myofibroblasts, resulting in production of a large amount of ECM. Upon activation, the HSCs presented with a myofibroblast-like phenotype: loss of cytoplasmic lipid droplets, and increased synthesis of type I and type III collagen.

Hepatic fibrosis is characterized by excessive deposition of collagens in the cavity of Disse. These collagens can be degraded by matrix metalloproteinase such as MMP-1. MMP-1 activity and its physiological inhibitor TIMP-1 interact with each other. In the process of hepatic fibrosis, MMP-1 expression is inhibited but TIMP1 expression increased, suggesting that the imbalance between MMP-1 and TIMP-1 plays an important role in the development of hepatic fibrosis. To increase collagenase activity and promote ECM degradation are important strategies for the clinical treatment of liver fibrosis. Studies have shown that [19], leptin may stimulate the synthesis of TIMP-1 in HSCs, which is dependent on peroxide $\left(\mathrm{H}_{2} \mathrm{O}_{2}\right)$ generation $\left(\mathrm{H}_{2} \mathrm{O}_{2}\right.$ can activate ERK1/2, p38, JAK1 and JAK2 signaling pathway), and may inhibit MMP-1 expression and increase type I collagen expression.

Our findings show leptin treatment could significantly reduce MMP-13 expression $(P<0.01)$, but had no influence on MMP-1 expression. Plumbagin treatment for $24 \mathrm{~h}$ also dramatically increased MMP-1 protein expression in HSC-LX2, but MMP-13 remained unchanged. Plumbagin can increase the MMP-1 expression, which is beneficial for ECM degradation. In addition, ELISA and western blot assays were performed to detect type I collagen expression in the supernatant of HSCs. ELISA revealed that treatment with plumbagin at different concentrations for $24 \mathrm{~h}$ inhibited the content of type I collagen in the supernatant. Western blot assay showed that, when compared with control group, $100 \mathrm{ng} / \mathrm{mL}$ leptin can stimulate the expression and secretion of type I collagen in the supernatant of HSCs, whereas plumbgin significantly inhibited it in HSCs. This suggests that plumbagin may significantly enhance collagen degradation, regulate the dynamic balance between ECM formation and degradation, reduce ECM deposition and decrease HSC activation, thereby restoring the 
normal function of HSCs. Thus, regulating collagen synthesis and degradation may be a promising approach to reverse liver fibrosis.

\section{CONCLUSION}

Plumbagin may inhibit leptin-induced proliferation of HSC-LX2 cells via blocking JAK-STAT3 and JAK-ERK1/2 signaling pathway, and exerting anti-fibrotic effects. Currently, there are no ideal anti-fibrotic drugs for clinical treatment of hepatic fibrosis. Our findings indicate that inhibiting HSC activation and proliferation at multiple targets and multiple levels may be beneficial for clinical prevention and treatment of liver fibrosis.

\section{ACKNOWLEDGEMENT}

This work was supported by the National Natural Science Foundation of China (nos. 81160553 and 81260675); Guangxi Education Foundation (no. 200103YB083); and University Foundation for the Talents of Guangxi Province (no. J11065).

\section{REFERENCES}

1. Priya S, Sudhakaran PR. Cell survival, activation and apoptosis of hepatic stellate cells: modulation by extracellular matrix proteins. Hepatol Res 2008; 38. 1221-1232.

2. Wang $P$, Liu $T$, Cong $M$, Wu $X$, Bai $Y$, Yin $C$, An $W$, Wang B, Jia J, You $H$. Expression of extracellular matrix genes in cultured hepatic oval cells: an origin of hepatic stellate cells through transforming growth factor beta? Liver Int 2009; 29: 575-584.

3. Bethanis SK, Theocharis SE. Leptin in the field of hepatic fibrosis: a pivotal or an incidental player? Dig Dis Sci 2006; 51: 1685-1696.

4. Handy JA, Fu PP, Kumar $P$, Mells JE, Sharma $S$, Saxena NK, Anania FA. Adiponectin inhibits leptin signalling via multiple mechanisms to exert protective effects against hepatic fibrosis. Biochem J 2011; 440: 385-395.

5. Handy JA, Saxena NK, Fu P, Lin S, Mells JE, Gupta NA Anania FA. Adiponectin activation of AMPK disrupts leptin-mediated hepatic fibrosis via suppressors of cytokine signaling (SOCS-3). J Cell Biochem 2010; 110: 1195-1207.

6. Yeh $\mathrm{HY}$, Klesius $\mathrm{PH}$. Over-expression, purification and immune responses to Aeromonas hydrophila ALO973 flagellar proteins. Fish Shellfish Immunol 2011; 31: 1278-1283.

7. Zhang $Y$, Proenca $R$, Maffei $M$, Barone $M$, Leopold $L$, Friedman JM. Positional cloning of the mouse obese gene and its human homologue. Nature 1994; 372: 425-432.
8. Shimamura $M$, Matsuda $M$, Ando $Y$, Koishi $R$, Yasumo $H$, Furukawa $H$, Shimomura I. Leptin and insulin down-regulate angiopoietin-like protein 3, a plasma triglyceride-increasing factor. Biochem Biophys Res Commun 2004; 322: 1080-1085.

9. Denver RJ, Bonett RM, Boorse GC. Evolution of leptin structure and function. Neuroendocrinology 2011; 94: 21-38.

10. Kanoski SE, Walls EK, Davidson TL. Interoceptive "satiety" signals produced by leptin and CCK. Peptides 2007; 28: 988-1002.

11. Suzukawa M, Nagase $H$, Ogahara I, Han K, Tashimo $H$, Shibui A, Koketsu R, Nakae S, Yamaguchi M, Ohta $K$. Leptin enhances survival and induces migration, degranulation, and cytokine synthesis of human basophils. J Immunol 2011; 186: 5254-5260.

12. Otte $C$, Otte JM, Strodthoff $D$, Bornstein SR, Folsch UR, Monig H, Kloehn S. Expression of leptin and leptin receptor during the development of liver fibrosis and cirrhosis. Exp Clin Endocrinol Diabetes 2004; 112: 10-17.

13. Wauman J, De Ceuninck L, Vanderroost $N$, Lievens $S$, Tavernier J. RNF41 (Nrdp1) controls type 1 cytokine receptor degradation and ectodomain shedding. J Cell Sci 2011; 124: 921-932.

14. Zhang $X$, Darnell JE, Jr. Functional importance of Stat3 tetramerization in activation of the alpha 2macroglobulin gene. J Biol Chem 2001; 276: 33576-33581.

15. Monaghan KA, Khong T, Burns CJ, Spencer A. The novel JAK inhibitor CYT387 suppresses multiple signalling pathways, prevents proliferation and induces apoptosis in phenotypically diverse myeloma cells. Leukemia 2011; 25: 1891-1899.

16. Machinal-Quelin F, Dieudonne MN, Leneveu MC, Pecquery R, Giudicelli Y. Proadipogenic effect of leptin on rat preadipocytes in vitro: activation of MAPK and STAT3 signaling pathways. Am J Physiol Cell Physiol 2002; 282: C853-863.

17. Saxena NK, Saliba G, Floyd JJ, Anania FA. Leptin induces increased alpha2(I) collagen gene expression in cultured rat hepatic stellate cells. J Cell Biochem 2003; 89: 311-320.

18. Tang $M$, Potter JJ, Mezey E. Leptin enhances the effect of transforming growth factor beta in increasing type I collagen formation. Biochem Biophys Res Commun 2002; 297: 906-911.

19. Cao Q, Mak KM, Ren C, Lieber CS. Leptin stimulates tissue inhibitor of metalloproteinase-1 in human hepatic stellate cells: respective roles of the JAK/STAT and JAK-mediated H2O2-dependant MAPK pathways. J Biol Chem 2004; 279: 42924304.

20. Saxena NK, Sharma D, Ding X, Lin S, Marra F, Merlin $D$, Anania $F A$. Concomitant activation of the JAK/STAT, PISK/AKT, and ERK signaling is involved in leptin-mediated promotion of invasion and migration of hepatocellular carcinoma cells. Cancer Res 2007; 67: 2497-2507. 